\title{
INVARIANTS OF DIFFERENTIAL CONFIGURATIONS IN THE
}

\author{
PLANE $^{*}$ \\ BY \\ EUGENE FRANCIS SIMONDS
}

INTRODUCTION

Suppose we have $n$ points $\left(x_{\nu}, y_{v}\right),(\nu=1,2,3, \cdots, n)$, in mutually general positions, $\dagger$ and let us assume that through each point $\left(x_{v}, y_{v}\right)$ there pass $\lambda_{\nu}$ regular analytic elements of order $r$. The elements belonging to any point may have contact of zero order-that is, merely intersect-or some of them may have contact with each other of any order less than $r$. The totality of the elements considered will be called a differential configuration of order $r$.

If such a differential configuration be subjected to a group of point transformations, it will be changed always into another of the same type, that is, having the same constants $n, \lambda_{\nu}$, and the order of contact of any two of the elements preserved. The numbers $n, \lambda_{\nu}$ are arithmetic invariants of the configuration under the group of point transformations, but will not necessarily be so under a group of contact transformations.

Rabut $\ddagger$ appears to have been the first to pay systematic attention to the differential invariants of such configurations. He considered two groups-the group of all point transformations in the plane, and that of all contact transformations in the plane. He claimed for his method that it gave not only all configurations having invariants-both relative and absolute-but also all possible types of such invariants.

Doubt was thrown on the generality of this method by Kasner, $\S$ who found (in another connection) a type of invariant which Rabut had declared impossible, and discussed a geometric interpretation of the same. (Rabut's actual error is pointed out by the writer in the present paper.)

\footnotetext{
* Presented to the Society, April 28, 1917.

† With regard to a group of transformations: the configuration of the points has no special property under the group.

$\ddagger$ Théorie des invariants universelles, Journal de l'ćcole polytechnique, ser. 2, vol. 4 (189S), pp. 137-203.

\$ The geometry of differential elements of the second order will respect to the group of all point transformations, A merican Journal of Mathematics, vol. 28 (1906), p. 210.
} 
In the article referred to Kasner also gave some invariants for the conformal group.*

The present paper deals only with the question of absolute invariants, more particularly with the types of configurations possessing them.

In Part I the problem is solved for any finite group of point transformations. It is shown that there are no essential invariants other than those of one or two elements. The invariants of the linear homogeneous group are found.

Part II treats of infinite groups of point transformations. In Section 1 it is shown that a single regular element has no invariant of order higher than zero. In Section 2 it is shown that every infinite group has invariants; but only for such configurations all of whose elements pass through a common point. The result admits of an exception, which is the subject of Section 3. In Section 4 we distinguish between groups involving arbitrary functions of only one variable, and those which involve at least one arbitrary function of two variables. Section 5 is devoted to a short consideration of the entire group of point transformations and of the area-preserving group. It is found that $2 n+2$ curves having contact of zero order have an invariant of order $n$ under the entire group. For the area-preserving group the number is $n+3$. Three types of invariants of lowest orders are given for each group. In Section 6 the equilong group is taken to illustrate Sections 3 and 4 . We find that three curves have a complete series of invariants-one of each orderand obtain a differential operator by which they are all derived from the first.

Part III is concerned with contact transformations. The results obtained for point transformations have their counterpart for the contact transformations. The exceptional groups corresponding to those of Section 3, Part II, are found to be reducible to the latter by contact transformations. Rabut's general theorem about the entire group of contact transformations is verified by examination of the equivalent complete system of infinitesimal transformations.

In general, the method employed is that of infinitesimal transformations, following the Lie theory.

The writer has great pleasure in thanking Professor Kasner for kind encouragement and helpful criticism.

\section{The invariants of Differential configurations in the Plane UNDER} FINITE GROUPS OF POINT TRANSFORMATIONS

We shall begin with a configuration of $n$ points such that all the $\lambda_{\nu}$ elements through any point $\left(x_{\nu}, y_{\nu}\right)$ have contact of zero order. Let the equation of

* Higher invariants of two elements for both the conformal and equilong groups are discussed by Kasner, Conformal Geometry, Proceedings of the fifth international congress, Cambridge (1912), vol. 2, pp. 81-87. See also other papers in these Transactions, vol. 16 (1915), pp. 333-349, and Bulletin of the American Mathematical Society, vol. 23 (1917), pp. 341-347. 
any element through $\left(x_{\nu}, y_{\nu}\right)$ be

$$
y_{\nu, j}=y_{\nu, j}\left(x_{\nu}\right) \quad\left(j=1,2,3, \cdots, \lambda_{\nu}\right) .
$$

Consider an $r$-parameter group of point-transformations

$$
\begin{aligned}
& X=\Phi\left(x, y, a_{1}, \cdots, a_{r}\right), \\
& Y=\Psi\left(x, y, a_{1}, \cdots, a_{r}\right),
\end{aligned}
$$

which does not degenerate in the neighborhood of the points $\left(x_{\nu}, y_{\nu}\right)$. Let the infinitesimal transformations of the group be

$$
X_{\rho} f \equiv \xi_{\rho}(x, y) \frac{\partial f}{\partial x}+\eta_{\rho}(x, y) \frac{\partial f}{\partial y} \quad(\rho=1,2, \cdots, r) .
$$

Under an infinitesimal transformation $X_{\rho} f$ all the quantities

$$
\begin{aligned}
& x_{\nu}, y_{\nu}, \cdots, y_{\nu, j}^{(k)} * \\
& \left(\nu=1,2, \cdots, n ; j=1,2, \cdots, \lambda_{\nu} ; k=1,2, \cdots ; r\right)
\end{aligned}
$$

are subjected to increments

$$
\begin{aligned}
& \delta x_{\nu}=\xi_{\rho}\left(x_{\nu}, y_{\nu}\right) \delta t, \\
& \delta y_{\nu}=\eta_{\rho}\left(x_{\nu}, y_{\nu}\right) \delta t \text {, } \\
& \delta y_{\nu, j}^{\prime}=\left\{\frac{\partial \eta_{\rho}\left(x_{\nu}, y_{\nu}\right)}{\partial x_{\nu}}+y_{\nu, j}^{\prime}\left(\frac{\partial \eta_{\rho}}{\partial y_{\nu}}-\frac{\partial \xi_{\rho}}{\partial x_{\nu}}\right)-{y_{\nu, j}^{\prime 2}}_{\partial \xi_{\rho}}^{\partial y_{\nu}}\right\} \delta t \equiv \eta_{\rho, \nu, j}^{\prime} \delta t, \\
& \delta y_{\nu, j}^{(k)}=\left\{\frac{d \eta_{\rho, \nu, j}^{(k-1)}}{d x_{\nu}}-y_{\nu, j}^{(k)} \frac{d \xi_{\rho}}{d x_{\nu}}\right\} \delta t \equiv \eta_{\rho, \nu, j}^{(k)} \delta t \\
& \left(\nu=1,2, \cdots, n ; j=1, \cdots, \lambda_{\nu} ; k=2,3, \cdots, r\right) .
\end{aligned}
$$

Any function $f\left(x_{\nu}, y_{\nu}, \cdots, y_{\nu j}^{(k)}, \cdots\right)$ is subjected to the increment $\left(X_{\rho}^{(r)} f\right) \delta t$, where

$$
X_{\rho}^{(r)} f \equiv \sum_{\nu=1}^{n} \xi_{\rho}\left(x_{\nu}, y_{\nu}\right) \frac{\partial f}{\partial x_{\nu}}+\sum_{\nu=1}^{n} \eta_{\rho} \frac{\partial f}{\partial y_{\nu}}+\sum_{\nu=1}^{n} \sum_{j=1}^{\lambda_{\nu}} \sum_{k=1}^{r} \eta_{\rho, \nu, j}^{(k)} \frac{\partial f}{\partial y_{\nu, j}^{(k)}} .
$$

The function $f\left(x_{\nu}, y_{\nu}, \cdots, y_{v, j}^{(k)}, \cdots\right)$ will be invariant under the group provided

$$
X_{\rho}^{(r)} f \equiv 0 \quad(\rho=1,2, \cdots, r) .
$$

Now by hypothesis the $r$ independent infinitesimal transformations generate the group (1). They therefore satisfy two by two relations of the form

$$
\begin{aligned}
\left(X_{i} X_{k}\right)=\sum_{s=1}^{r} c_{i k s} X_{s} f \\
\quad\left(i, k=1,2, \cdots, r ; c_{i k s}=\text { constant }\right) .
\end{aligned}
$$

* We denote by $f^{(k)}$ the $k$ th derivative of $f(x)$. 
It is well known that the extended infinitesimal transformations $X_{\rho}^{(r)} f$ satisfy the analogous relations

$$
\left(X_{i}^{(r)} X_{k}^{(r)}\right)=\sum_{s=1}^{r} c_{i k s} X_{s}^{(r)} f
$$

and generate an $r$-parameter group in the variables $x_{\nu}, y_{v}, \cdots, y_{v j}^{k}, \cdots$.

When equated to zero the expressions (2) form a complete system of $r$ partial differential equations in the $2 n+r \sum \lambda_{\nu}$ variables $x_{\nu}, y_{\nu}, \cdots, y_{v, j}^{(k)}, \cdots$. From the theory of complete systems we know that the number of independent solutions is

$$
2 n-r+r \sum_{\nu=1}^{n} \lambda_{\nu}
$$

These solutions are independent invariants of our differential configuration under the group of point transformations.

Of these invariants a certain number may be accounted for by taking them to be the ordinary differential invariants of single elements of the configuration. Each element has two of order not greater than $r-J_{r}$ and $J_{r-1}$; the former is of order $r$, the latter of order $k(<r)$. If the group is transitive $k>0$; if it is intransitive $k=0$. The remaining independent invariants must then involve at least two elements. We shall refer to them as "mixed invariants." The number in the case of an $r$-parameter transitive group is

$$
N_{T}=(2 n-r)+(r-2) \sum \lambda_{\nu},
$$

and in the case of an intransitive group

$$
N_{I}=(n-r)+(r-1) \sum \lambda_{\nu} .
$$

We have so far supposed the elements through each of the $n$ points to have contact of zero order. If this is not so let us assume the number of elements through the point $\left(x_{v}, y_{v}\right)$ when the highest order considered is $\lambda$ to be $n_{v, \lambda}$. The number of variables is now

$$
2 n+\sum_{\nu=1}^{n} \sum_{\lambda=1}^{r} n_{\nu, \lambda} .
$$

The number of independent solutions is consequenty

$$
(2 n-r)+\sum_{\nu=1}^{n} \sum_{\lambda=1}^{r} n_{\nu, \lambda} .
$$

For a transitive group the number of ordinary differential invariants will be

$$
\sum_{\nu=1}^{n}\left(n_{\nu, r}+n_{\nu, k}\right)
$$

where $k$ is the order of $J_{r-1}$; in this case, the number of mixed invariants is 


$$
N=(2 n-r)-\sum_{\nu=1}^{n}\left(n_{\nu, r}+n_{\nu, k}\right)+\sum_{\nu=1}^{n} \sum_{\lambda=1}^{r} n_{\nu, \lambda} .
$$

For an intransitive group we replace $\sum n_{\nu, k}$ by $n$.

The difference between this number $N$ and the number of essential mixed invariants of the $n$ "bunches" considered separately will be the number of independent essential invariants involving elements from more than one bunch. The latter is

$$
n(2-r)-\sum_{\nu=1}^{n}\left(n_{\nu, r}+n_{\nu, k}\right)+\sum_{\nu=1}^{n} \sum_{\lambda=1} n_{\nu, \lambda} .
$$

The difference is therefore $(n-1) r$, whether the group be transitive or not. Now two elements chosen fiom different bunches have $r$ independent mixed invariants; hence the $(n-1) r$ invariants may clearly be taken to be those of $n-1$ configurations of two elements only, the elements of a pair being chosen from different bunches. It follows that the only essential invariants other than those of individual bunches may be taken to be those of two elements.

Let the first-order elements of a bunch through the point $(x, y)$ be denoted by $y_{1}^{\prime}, y_{2}^{\prime}, \cdots, y_{m_{1}}^{\prime}$, and let the number of elements of order $r$ having the common slope $y_{i}^{\prime}$ be $n_{i}^{(i)}$. The configuration consisting of these elements alone has, say, $N_{i}^{(0)}$ invariants, whereas the whole bunch has $N$. We have, then,

$$
\begin{aligned}
\sum N_{i}^{(0)} & =V-m_{1} r+2\left(m_{1}-1\right), \\
N & =V-r,
\end{aligned}
$$

where $V$ is the number of variables in the whole bunch. Subtracting, we have

$$
N-\sum N_{i}^{(\theta)}=\left(m_{1}-1\right)(r-2) .
$$

If the group is transitive, these $\left(m_{1}-1\right)(r-2)$ invariants are the independent invariants of any set of $m_{1}$ elements of order $r$, and may be taken to be the $m_{1}-1$ sets of $(r-2)$ independent invariants of configurations consisting of pairs of elements having contact of order zero. If the group is intransitive the equation (3) takes the form

$$
N-\left(\sum N_{i}^{(0)}-\overline{m-1}\right)=\left(m_{1}-1\right)(r-1),
$$

showing that there are $\left(m_{1}-1\right)(r-1)$ invariants of the above type. All other invariants of the bunch can be taken to be invariants of one or other of the sets $N_{i}^{(0)}$, that is, of configurations all of whose elements have contact of at least the first order.

Continuing the method of exhaustion for bunches all of whose elements have contact of the first order, we find in the same way that there are no essential 
invariants of elements having contact of the first order other than those of pairs of elements.

And in general, if $y_{1}^{(s)}, y_{2}^{(s)}, \cdots, y_{m_{\mathrm{e}}}^{(s)}$ be the only distinct elements of order $s$ of the bunch, and if $N_{i}^{(s)}$ be the number of independent invariants of the configuration having the common element $y_{i}^{(s)}$ we have

$$
N-\sum N_{i}^{(s)}=\left(m_{s}-1\right)(r-s-2) .
$$

In the case of a group where the order $k$ of $J_{r-1}$ is greater than $s$, the number $\left(m_{s}-1\right)(r-s-2)$ is the number of independent invariants of $m_{s}$ elements of order $r$ having contact of order $s$, exclusive of the types $J_{r}$ and $J_{r-1}$. These invariants may be taken to be those of pairs of elements. If $k \leqq s$, instead of $\sum N_{i}^{(s)}$ we must subtract $\sum N_{i}^{(s)}-\overline{m_{\varepsilon}-1}$ from $N$, and we get in that case $\left(m_{s}-1\right)(r-s-1)$ invariants of pairs of elements having contact of order $s$.

We have, then, the following result for elements of order $\ngtr r$ :

Under an r-parameter group of point transformations in the plane there are no essential invariants of order less than or equal to $r$ other than those belonging to configurations of one or two elements.

So far we have spoken of elements of order $\ngtr r$. If we consider all elements of order $r+r^{\prime}$, the number of additional independent invariants of any of the configurations considered can be accounted for by taking them to be the invariants of single elements, of which each curve has $r^{\prime}$ in addition to $J_{r}$ and $J_{r-1}$. They are

$$
J_{r+1}=\frac{d J_{r}}{d J_{r-1}}, \quad J_{r+2}=\frac{d J_{r+1}}{d J_{r}}, \quad \cdots, \quad J_{r+r^{\prime}}=\frac{d J_{r+r^{\prime}-1}}{d J_{r+r^{\prime}-2}} .
$$

The final statement for all configurations is as follows:

An r-parameter group of point transformations in the plane has no essential mixed invariants other than those of two elements.

It will be convenient to distinguish symbolically the different types of configurations of two elements. We represent by $(1: 1)_{r}$ that consisting of two distinct points $\left(x_{1}, y_{1}\right)$ and $\left(x_{2}, y_{2}\right)$ with an element of order $r$ through each. The configuration (2): is that one consisting of two elements of order $r$ through a single point $(x, y)$, the elements having contact of order $s$. If the elements have contact of zero order the symbol is $(2)_{r}^{0}$. A single element of order $r$ is represented by $(1)_{r}$.

We may illustrate the foregoing by reference to the linear homogeneous group

$$
X=a_{1} x+a_{2} y, \quad Y=a_{3} x+a_{4} y .
$$

The four mixed invariants of $(1: 1)_{4}$ are 


$$
\begin{aligned}
I_{1}=\frac{y_{2}-x_{2} y_{1}^{\prime}}{y_{1}-x_{1} y_{1}^{\prime}} ; & I_{2}=\frac{y_{2}-x_{2}}{y_{1}-x_{1}} \frac{y_{2}^{\prime}}{y_{2}^{\prime}} ; \\
I_{3}=\frac{y_{1}^{\prime \prime}\left(x_{1} y_{2}-x_{2} y_{1}\right)^{2}}{\left[\left(y_{2}-y_{1}\right)-\left(x_{2}-x_{1}\right) y_{1}^{\prime}\right]^{3}} ; & I_{4}=\frac{y_{2}^{\prime \prime}\left(x_{1} y_{2}-x_{2} y_{1}\right)^{2}}{\left[\left(y_{2}-y_{1}\right)-\left(x_{2}-x_{1}\right) y_{2}^{\prime}\right]^{3}} .
\end{aligned}
$$

We can obtain the two mixed invariants of $(2)_{4}^{0}$ from the above by seeking a function $f\left(I_{1}, I_{2}, I_{3}, I_{4}\right)$ which shall be independent of $x_{2}, y_{2}$. Such a function, if it exists, will be a solution of the pair of equations

$$
\sum \frac{\partial f}{\partial I_{i}} \frac{\partial I_{i}}{\partial x_{2}}=0, \quad \sum \frac{\partial f}{\partial I_{i}} \frac{\partial I_{i}}{\partial y_{2}}=0
$$

Two independent solutions are

$$
\begin{aligned}
& I_{1}^{(0)}=\frac{\left(I_{1}-I_{2}\right)^{2}}{I_{3}\left(I_{1}-1\right)^{3}}=\frac{y_{1}^{\prime \prime}\left(y_{1}-x_{1} y_{2}^{\prime}\right)^{2}}{\left(y_{1}^{\prime}-y_{2}^{\prime}\right)^{2}} \frac{\left(y_{1}-x_{1} y_{1}^{\prime}\right)}{} \\
& I_{2}^{(0)}=\frac{\left(I_{1}-I_{2}\right)^{2}}{I_{4}\left(I_{2}-1\right)^{3}}=\frac{y_{2}^{\prime \prime}\left(y_{1}-x_{1} y_{1}^{\prime}\right)^{2}}{\left(y_{1}^{\prime}-y_{2}^{\prime}\right)^{2}\left(y_{1}-x_{1} y_{2}^{\prime}\right)} .
\end{aligned}
$$

Finally, to obtain the unique mixed invariant of $(2)_{4}^{0}$, we divide $I_{1}^{(0)}$ by $I_{2}^{(0)}$ to eliminate the factor $y_{1}^{\prime}-y_{2}^{\prime}$, and put $y_{2}^{\prime}=y_{1}^{\prime}$. The result is

$$
I_{1}^{(1)}=\frac{y_{1}^{\prime \prime}}{y_{2}^{\prime \prime}}
$$

The types $I_{1}, I_{2}, I_{3}, I_{4} ; I_{1}^{(0)}, I_{2}^{(0)} ; I_{1}^{(1)}$ are the only essential ones belonging to the linear homogeneous group apart from the series of invariants of a single element.

\section{INFINITE GROUPS OF POINT TRANSFORMATIONS}

We consider the same problem when our group of transformations, viz.,

$$
X=\Phi(x, y), \quad Y=\Psi(x, y),
$$

is infinite. The infinitesimal transformations of such a group will be of the form

$$
\xi(x, y) \frac{\partial f}{\partial x}+\eta(x, y) \frac{\partial f}{\partial y}
$$

where $\xi$ and $\eta$ are solutions of a system of linear partial differential equations*

$$
A_{i} \xi+B_{i} \eta+C_{i} \xi_{x}+D_{i} \xi_{y}+E_{i} \eta_{x}+F_{i} \eta_{y}+\cdots=0
$$

The general solution of this system involves one or more arbitrary functions either of one variable or of two. The most general case is that of the whole

*Lie, Mathematische Annalen, vol. 24 (1884), p. 553. 
group of point transformations; the system (2) reduces to the identity $0=0$ and both $\xi$ and $\eta$ are arbitrary functions of $x$ and $y$.

If it be required to find the differential invariants of the group (1), say of order $n$, we begin by "extending" the infinitesimal transformations, i. e., we calculate the increments of $y^{\prime}, y^{\prime \prime}, \cdots, y^{(n)}$. Let us call them, as is usual, $\eta^{\prime} \delta t, \eta^{\prime \prime} \delta t, \cdots, \eta^{(n)} \delta t$. We give below such terms as are important in what is to follow.

$$
\begin{aligned}
& \eta^{\prime}=\eta_{x}+\left(\eta_{y}-\xi_{x}\right) y^{\prime}-\xi_{y}{y^{\prime 2}}^{2} \\
& \eta^{\prime \prime}=\eta_{x x}+\left(2 \eta_{x y}-\xi_{x x}\right) y^{\prime}+\left(\eta_{y y}-2 \xi_{x y}\right){y^{\prime}}^{2}-\xi_{y y}{y^{\prime}}^{3} \\
& +\left(\eta_{y}-2 \xi_{x}\right) y^{\prime \prime}-3 \xi_{y} y^{\prime} y^{\prime \prime}, \\
& \eta^{(n)}=\left\{\eta_{n, 0}+\sum_{k=1}^{n}\left(c_{k} \eta_{n-k, k}-c_{k-1} \xi_{n-k+1, k-1}\right) y^{\prime k}-\xi_{0, n} y^{\prime n+1}\right\} \\
& +\left\{\sum_{k=1}^{n-1} k\left(\bar{c}_{k} \eta_{n-k-1, k}-\bar{c}_{k-1} \xi_{n-k, k-1}\right) y^{\prime k-1} y^{\prime \prime}-n \xi_{0, n-1} y^{\prime n-1} y^{\prime \prime}\right\} \\
& +\left\{\left(\eta_{n-2,1}-2 \xi_{n-1,0}\right) y^{\prime \prime}+\left(\overline{n-2} \eta_{n-3,2}\right.\right. \\
& \left.-\overline{2 n-1} \xi_{n-2,1}\right) y^{\prime} y^{\prime \prime}+\cdots \\
& \left.+\left(2 \eta_{0, n-1}-3 \overline{n-1} \xi_{1 n-2}\right) y^{\prime n-2} y^{\prime \prime}-3 \xi_{0, n-1} y^{\prime n-1} y^{\prime \prime}\right\}+\cdots .
\end{aligned}
$$

Here $f_{n-k, k}$ is the derivative of the $n$th order obtained by differentiating $f(x, y) n-k$ times with respect to $x$, and $k$ times with respect to $y ; c_{k}$ is the coefficient of $x^{k}$ in the expansion of $(1-x)^{n}$; and $\bar{c}_{k}$ is the coefficient of $x^{k}$ in the expansion of $(1-x)^{n-1}$. In the expression for $\eta^{(n)}$, three sets of terms are given. The first is a polynomial in $y^{\prime}$, whose coefficients involve all the partial derivatives of $\xi$ and $\eta$ of order $n$. The coefficients in the second already occur in $\eta^{(n-1)}$; the third involves $n-1$ functions of the derivatives of order $n-1$ which are all independent of those already occurring in $\eta^{(n-1)}$. The three sets of terms contain all the terms of $\eta^{(n)}$ whose coefficients involve derivatives of $\xi$ and $\eta$ of orders $n-1$ and $n$.

Any function $f\left(x, y, \cdots, y^{(n)}\right)$ is subjected by the infinitesimal transformation $\xi, \eta$ to an increment

$$
\left\{\xi \frac{\partial f}{\partial x}+\eta \frac{\partial f}{\partial y}+\eta^{\prime} \frac{\partial f}{\partial y^{\prime}}+\cdots+\eta^{(n)} \frac{\partial f}{\partial y^{(n)}}\right\} \delta t,
$$

where $\xi$ and $\eta$ are subject to the conditions imposed by the defining system (2). Now the equations of this system enable us to express certain of the functions $\xi, \eta, \xi_{x}, \xi_{y}, \eta_{x}, \eta_{y}, \cdots$ in terms of the rest. Making the resulting substitutions we reduce the number of independent functions occurring in the ex- 
pressions $\xi, \eta, \eta^{\prime}, \eta^{\prime \prime}, \cdots, \eta^{(n)}, \ldots$. The system (3) is then equivalent to a complete system of, say, $N^{(n)}$ infinitesimal transformations

$$
\alpha_{j} \frac{\partial f}{\partial x}+\beta_{j} \frac{\partial f}{\partial y}+\cdots+\beta_{j}^{(n)} \frac{\partial f}{\partial y^{(n)}} \quad\left(j=1,2, \cdots, N^{(n)}\right),
$$

where $\alpha_{j}, \beta_{j}$ are functions of $x$ and $y$, and $\beta_{j}^{(n)}$ is a function of $x, y, y^{\prime}, \cdots, y^{(n)}$. If an element of order $n$ has invariants under the group, they will be solutions of the equations obtained by setting the expressions (4) equal to zero.

1. A single regular element. Considering first a single element, we know that the system sometimes has a solution of order zero. For example if $\alpha_{j}=0$, a solution will be $x$. The largest group with this invariant is

$$
X=x, \quad Y=\Psi(x, y),
$$

$\Psi$ being an arbitrary function.

We are further able to assert the following:

No infinite group of point transformations can have an invariant of a single element of order greater than zero.

This result is implicit in the following theorem of Lie's:* "If an ordinary differential invariant of the $r$ th order 'fixes' a $\rho$-parameter group of transformations, the number $\rho$ has a finite upper limit."

For, by equating to a constant a differential invariant of order $n$, we get a differential equation of order $n$ which is invariant. Thus, there is no infinite group with an invariant of order $>1$. Moreover, if the group have an invariant $f\left(x, y, y^{\prime}\right)$, it will leave invariant the differential equation

$$
f_{x}+f_{y} y^{\prime}+f_{y^{\prime}} y^{\prime \prime}=0,
$$

and that in a special way, for it will leave invariant each of the $\infty^{1}$ families $f\left(x, y, y^{\prime}\right)=c$ of the $\infty^{2}$ integral-curves. Thus Lie's theorem excludes invariants of order 1 .

The following is an independent proof, more in line with the other considerations of this chapter. Let

$$
X=\Phi(x, y), \quad Y=\Psi(x, y)
$$

be a group of point transformations, and let

$$
U f \equiv \xi \frac{\partial f}{\partial x}+\eta \frac{\partial f}{\partial y}
$$

be an infinitesimal transformation of the group. $\xi$ and $\eta$ are solutions of a system of defining-equations. Suppose the group to have a differential invariant of order $n(>0)$ e. g.,

$$
\Omega\left(x, y, y^{\prime}, \cdots, y^{(n)}\right) .
$$

* Lie-Scheffers, Continuierliche Gruppen, Kap. 12, § 1.

Trans. Am. Math. Soc. 16 
Then it is necessary and sufficient that

$$
U^{(n)} \Omega=0,
$$

where $U^{(n)} f$ is the $n$-fold extension of $U f . U^{(n)} f$ is, in fact,

$$
\xi \frac{\partial f}{\partial x}+\eta \frac{\partial f}{\partial y}+\eta^{\prime} \frac{\partial f}{\partial y^{\prime}}+\cdots+\eta^{(n)} \frac{\partial f}{\partial y^{(n)}},
$$

where the expressions for $\eta^{\prime}, \eta^{\prime \prime}, \cdots, \eta^{(n)}$ are those given by equations (3).

We must have, then, for all values of $y^{\prime}, y^{\prime \prime}, \cdots, y^{(n)}$,

$$
U^{(n)} \Omega=0,
$$

in virtue of the relations between $\xi$ and $\eta$ and their partial derivatives given by the defining-equations of the group.

Conversely, the defining-equations of the largest group having the invariant $\Omega$ are obtained from the identity

$$
U^{(n)} \Omega=0
$$

in the variables $y^{\prime}, y^{\prime \prime}, \cdots, y^{(n)}$, by equating to zero the coefficients of the different powers of $y^{\prime}, y^{\prime \prime}, \cdots, y^{(n)}$ and their products.

Solving the equation (5) for $\eta^{(n)}$ we have

$$
\eta^{(n)}=-\left(\xi \frac{\partial \Omega}{\partial x}+\eta \frac{\partial \Omega}{\partial y}+\cdots+\eta^{(n-1)} \frac{\partial \Omega}{\partial y^{(n-1)}}\right) \div \frac{\partial \Omega}{\partial y^{(n)}} .
$$

This is an identity in $y^{\prime}, y^{\prime \prime}, \cdots, y^{(n)}$. Let us now choose such a set of values for $y^{\prime \prime}, \cdots, y^{(n)}$ that $\partial \Omega / \partial y^{(n)}$ does not vanish. After substitution we have, making use of the expression for $\eta^{(n)}$ in equations (3),

$$
\begin{aligned}
\eta_{n, 0}+\left(c_{1} \eta_{n-1,1}-\xi_{n, 0}\right) y^{\prime}+\sum_{k=2}^{n}\left(c_{k} \eta_{n-k, k}-\right. & \left.c_{k-1} \xi_{n-k+1, k-1}\right) y^{\prime k}+\cdots \\
& +c_{n} \xi_{0, n} y^{\prime n+1}=\lambda\left(x, y, y^{\prime}\right),
\end{aligned}
$$

where $\lambda$ involves partial derivatives of $\xi$ and $\eta$ of order lower than $n$. This is an identity in $y^{\prime}$. If we choose $n+2$ different values of $y^{\prime}$, no one of which causes the denominator of $\lambda$ to vanish, we obtain a system of $n+2$ linear independent equations in the $n+2$ quantities

$$
\eta_{n, 0}, c_{k} \eta_{n-k, k}-c_{k-1} \xi_{n-k+1, k-1}, \xi_{0, n} .
$$

Solving, we get $n+2$ equations of the type

$$
\begin{aligned}
\eta_{n, 0} & =\sigma_{0}, \\
c_{k} \eta_{n-k, k}-c_{k-1} \xi_{n-k+1, k-1} & =\sigma_{k} \quad(k=1,2, \cdots, n), \\
\xi_{0, n} & =\sigma_{n+1},
\end{aligned}
$$


where the $\sigma$ 's are functions of the partial derivatives of $\xi$ and $\eta$ of order lower than $n$, and also perhaps involve $x$ and $y$ explicitly.

If we differentiate these equations partially for $x$ and $y$ we get a system of $2(n+2)$ linear equations

$$
\begin{aligned}
\left(\eta_{n, 0}\right)_{x} & =\left(\sigma_{0}\right)_{x}, \\
\left(\eta_{n, 0}\right)_{y} & =\left(\sigma_{0}\right)_{y}, \\
\left(c_{k} \eta_{n-k, k}-c_{k-1} \xi_{n-k+1, k-1}\right)_{x} & =\left(\sigma_{k}\right)_{x}, \quad(k=1,2, \cdots, n), \\
\left(c_{k} \eta_{n-k, k}-c_{k-1} \xi_{n-k+1, k-1}\right)_{y} & =\left(\sigma_{k}\right)_{y}, \\
\left(\xi_{0, n}\right)_{x} & =\left(\sigma_{n+1}\right)_{x}, \\
\left(\xi_{0, n}\right)_{y} & =\left(\sigma_{n+1}\right)_{y},
\end{aligned}
$$

in the $2(n+2)$ partial derivatives of $\xi$ and $\eta$ of order $n+1$, whose determinant has the value

$$
(-1)^{n}\left(c_{1}-c_{1}^{2}\right)\left(c_{1} c_{3}-c_{2}^{2}\right) \cdots\left(c_{n+2} c_{n}-c_{n+1}^{2}\right)
$$

and hence does not vanish. The system consisting of (6) and (7) together has the following properties:

(a) The highest order of the partial derivatives of $\xi$ and $\eta$ occurring in the system being $n+1$, it is possible to express all the derivatives of order $n+1$ in terms of those of lower orders, and this is not the case for all the derivatives of any order less than $n+1$.

(b) It is not possible through partial differentiation of the system and combination of the resulting equations with each other and with those of the system to obtain any further equations expressing relations between the derivatives from the first to the $n$th order, independent of those already occurring in the system.

Now any system of partial differential equations possessing these two properties is integrable, and its general solution involves only a finite number of arbitrary constants.*

The whole system of defining-equations - of which the system (6) is but a part-will therefore have a solution-pair $\xi, \eta$, involving only a finite number of parameters.

The group with the invariant $\Omega$ is therefore finite.

2. Configurations which have invariants. Turning now to the differential configuration consisting of $r$ distinct points $\left(x_{\nu}, y_{\nu}\right)$, the number of elements in the "bunch" (if we may adopt the word) through $\left(x_{\nu}, y_{\nu}\right)$ being $\lambda_{\nu}$, we see at once a fundamental distinction between finite and infinite groups. The set of essential arbitrary functions $\xi, \eta, \xi_{x}, \eta_{x}, \xi_{y}, \eta_{y}, \cdots$ to be elimi-

* Lie, Theorie der Transformationsgruppen, vol. 1, Kap. 10. 
nated occurs in each bunch with different arguments. Each bunch, therefore, has its own complete system, and the complete system belonging to the whole configuration is merely the combination of the complete systems of the individual bunches. Since the same is obviously true of the number of variables in the whole configuration, we have the following

THEOREM. The only essential invariants, under an infinite group of point transformations, of a differential configuration consisting of several bunches of elements whose base-points are in mutually general positions are those of the individual bunches.

We have to consider, then, the configuration $(\lambda)_{n}^{0}$ consisting of $\lambda$ elements of order $n$ having the point $(x, y)$ in common. The equivalent complete system is

$$
\begin{aligned}
\alpha_{j}(x, y) \frac{\partial f}{\partial x}+\beta_{j}(x, y) \frac{\partial f}{\partial y} & +\sum_{k=1}^{n} \beta_{j \mu}^{(k)} \frac{\partial f}{\partial y_{\mu}^{(k)}} \\
& \left(j=1,2, \cdots, N_{n} ; \mu=1,2, \cdots, \lambda\right) .
\end{aligned}
$$

For $\lambda=1$, there is no invariant of order greater than zero, but since the number of equations in the complete system depends on the group, while $\lambda$ is at our disposal, it is clearly possible by sufficiently increasing $\lambda$ to obtain a configuration which shall have an invariant of order $n$.

The theorem that all groups of point-transformations have invariants of certain configurations is a result due to Rabut,* who also showed that the same was true for all groups of contact transformations.

3. Certain imprimitive groups. We have seen that an infinite group of point transformations has no essential invariants apart from those of individual bunches, provided the base-points of the bunches are in mutually general positions. In the case, however, of certain imprimitive groups if we choose the base-points $\left(x_{\nu}, y_{v}\right)$ of the bunches on the same integral-curve of the invariant differential equation of the first order, it happens that there are essential invariants of configurations consisting of two or more bunches. All imprimitive groups of the plane are equivalent under the group of point transformations to the group

$$
X=\Phi(x), \quad Y=\Psi(x,) .
$$

We may ask for the most general form of the function $\Psi$ so that the group may have invariants of the above type.

It is clear that the most general configuration of the desired type is that consisting of, say, $r$ distinct points $\left(x, y_{\nu}\right)$ each with a single element passing through it. Let the infinitesimal transformations of the group be

$$
\delta x=\xi(x) \delta t ; \quad \delta y=\dot{\eta}(x, y) \delta t .
$$

* I.oc. cit. 
Now $\eta$ can involve no arbitrary function of $y$, which varies from element to element, since this would necessarily lead to the equations

$$
\frac{\partial f}{\partial y_{\nu}}=0, \quad \frac{\partial f}{\partial y_{\nu}^{\prime}}=0, \quad \cdots, \quad \frac{\partial f}{\partial y_{\nu}^{(k)}}=0 ;
$$

$\eta$ then, must be a linear expression of the form

$$
\alpha_{1}(y) \beta_{1}(x)+\alpha_{2}(y) \beta_{2}(x)+\cdots+\alpha_{k}(y) \beta_{k}(x),
$$

where the $\alpha$ 's are definite functions of $y$, but the $\beta$ 's may be arbitrary functions of $x$. The equivalent complete system of the unextended group is then

$$
\frac{\partial f}{\partial x}, \alpha_{k} \frac{\partial f}{\partial y} \quad(k=1,2, \cdots) .
$$

Since these generate a finite group, the transformations

$$
\alpha_{k} \frac{\partial f}{\partial y} \quad(k=1,2, \cdots),
$$

themselves generate a finite group in the variable $y$. There are, however, only three independent infinitesimal transformations in one variable generating a finite group viz.,

so that $\eta$ is of the form

$$
\frac{\partial f}{\partial y}, y \frac{\partial f}{\partial y}, y^{2} \frac{\partial f}{\partial y}
$$

$$
\beta_{1}(x)+y \beta_{2}(x)+y^{2} \beta_{3}(x) .
$$

Then the finite equations of the group are

$$
X=\Phi(x), \quad Y=\frac{\Psi_{1}(x)+\Psi_{2}(x) y}{\Psi_{3}(x)+\Psi_{4}(x) y},
$$

$\Phi, \Psi_{1}, \Psi_{2}, \Psi_{3}, \Psi_{4}$ being arbitrary functions of $x$, only three of the last four, of course, being essential.

This group has an invariant of four points having the same abscissa, viz.,

$$
\frac{\left(y_{1}-y_{2}\right)\left(y_{3}-y_{4}\right)}{\left(y_{2}-y_{3}\right)\left(y_{4}-y_{1}\right)} \text {. }
$$

4. Number of elements having invariants. There is also a distinction between groups whose equations involve arbitrary functions of two variables and those involving only arbitrary functions of one variable. In the former case, the number of " new" arbitrary functions after each successive differentiation is an increasing function of $n$. If $\lambda_{n}$ be the lowest number of curves (having contact of order zero) with an invariant of order $n$, we have in succession (expressing algebraically the fact that the number of independent 
solutions of a complete system is equal to the number of variables minus the number of independent equations)

$$
\begin{aligned}
\lambda_{1} & =N_{1}+1, \\
\lambda_{2}+\lambda_{1} & =N_{2}+2, \\
\lambda_{3}+\lambda_{2}+\lambda_{1} & =N_{3}+3, \\
\cdot \quad \cdot \cdot \cdot \cdot \cdot & \cdot \cdot \cdot \cdot \\
\lambda_{n}+\lambda_{n-1}+\cdots+\lambda_{1} & =N_{n}+n .
\end{aligned}
$$

Subtracting the last two we get

$$
\lambda_{n}=1+\left(N_{n}-N_{n-1}\right),
$$

which shows that $\lambda_{n}$ is an increasing function of $n$. In the second case, $N_{n}-N_{n-1}$ is constant, and equal to the number of unrelated arbitrary functions in the unextended form of the group. From the category of functions which are " unrelated" to a given set of functions, we must exclude not only dependent functions, but those dependent on the given set and their derivatives. If the number of unrelated functions in the group is $k, k+1$ curves have an invariant of each order after the lowest.

It was assumed in the foregoing that the curves of the configuration had contact of the lowest possible order, that is to say, that in the case of the groups considered in Section 3 all the elements pass through points which have but one coördinate in common, and in all other cases have contact of zero order. We may say then:

TheoRem. If the elements of our configuration have contact of the lowest possible order consistent with the existence of differential invariants, and if $\lambda_{n}$ be the lowest number of elements having an invariant of order $n$ under an infinite group, two cases occur:

(a) $\lambda_{n}$ is an increasing function of $n$ if the group involves at least one arbitrary function of two variables;

(b) $\lambda_{n}$ attains a final value $k+1$ if the equations of the group involve $k$ arbitrary functions all of one variable.

5. The group of all point-transformations and the group which preserves area. As an example illustrating Section 2 and the first case of Section 4, we take the area-preserving group; to illustrate Section 3 and the second case of Section 4 we discuss the equilong group. First, however, we obtain a theorem about the entire group of point transformations.

The theory of this group was partly worked out by Rabut,* who obtained a set of results which he supposed to be perfectly general and all-inclusive, but which were shown to be not so by Kasner. $\dagger$ Among other correct results

${ }^{*}$ Loc. cit.

$\dagger$ Loc. cit. 
Rabut shows $(a)$ the invariants are free from $x$ and $y$; $(b)$ each bunch must be considered separately; $(c)$ the invariants are of degree and of weight zero in all of the derivatives; $(d)$ derivatives of the same order appear only in their differences two by two. He goes on, however, to say: "in order that $y_{a}^{(m)}$ $-y_{b}^{(m)}$ may occur in an invariant, the curves $a$ and $b$ must have contact of order $m-1 . "$ The proof given* is fallacious, as the invariant obtained by Kasner showed. This error made an apparent simplification of the problem, whose complete solution, in fact, is not practicable, owing to the increasing complexity of the expressions for the successive derivatives of $Y$ with regard to $X$. This (the chief obstacle to a complete solution of the problem) was entirely avoided by Rabut's erroneous assumption. His results, then, are far from being general, but apply only to a very limited class of configurations.

In equations (3) we found expressions for the increments of the variables $x, y, y^{\prime}, \cdots, y^{(n)}$ under the general infinitesimal transformation of the entire group, pointing out what arbitrary independent functions of $x$ and $y$ occurred in the various expressions. The number of these is $n(n+2)+2$. Thus the general infinitesimal transformation of the group is equivalent to a complete system of $n(n+2)+2$ infinitesimal transformations of which the first two are $\partial f / \partial x, \partial f / \partial y$. The formula (8) gives $\lambda_{n}=2 n+2$, and the complete system of equations for a bunch of $2 n+2$ curves intersecting in a common point, is as follows:

$$
\begin{aligned}
& \frac{\partial f}{\partial x}=0 ; \quad \frac{\partial f}{\partial y}=0 ; \quad \sum_{\imath=1}^{2 n+1} \frac{\partial f}{\partial y_{\imath}^{\prime}}=0, \\
& \sum_{\imath=1}^{2 n+2}\left(y_{\imath}^{\prime} \frac{\partial f}{\partial y_{\imath}^{\prime}}+y_{\imath}^{\prime \prime} \frac{\partial f}{\partial y_{\imath}^{\prime \prime}}+\cdots+y_{\imath}^{(n)} \frac{\partial f}{\partial y_{\imath}^{n}}\right)=0, \\
& \sum_{\imath=1}^{2 n+2}\left(y_{\imath}^{\prime} \frac{\partial f}{\partial y_{\imath}^{\prime}}+2 y_{\imath}^{\prime \prime} \frac{\partial f}{\partial y_{\imath}^{\prime \prime}}+\cdots+n y_{\imath}^{(n)} \frac{\partial f}{\partial y_{\imath}^{(n)}}\right)=0, \\
& \sum_{\imath=1}^{2 n+2}\left(y_{\imath}^{\prime 2} \frac{\partial f}{\partial y_{\imath}^{\prime}}+3 y_{\imath}^{\prime} y_{\imath}^{\prime \prime} \frac{\partial f}{\partial y_{\imath}^{\prime \prime}}+\cdots\right)=0, \\
& \sum_{\imath=1}^{2 n+2} y_{\imath}^{\prime \mu} \frac{\partial f}{\partial y_{\imath}^{(n)}}=0 \quad(\mu=0,1,2, \cdots, n+1), \\
& \sum_{\imath=1}^{2 n+2} y_{\imath}^{\prime \nu} y_{\imath}^{\prime \prime} \frac{\partial f}{\partial y_{\imath}^{(n)}}=0 \quad(\nu=0,1,2, \cdots, n-2) .
\end{aligned}
$$

The first two of these equations show that $x$ and $y$ do not occur in an invariant, as already stated.

We must justify our use of the formula (8) by showing that the equations

*P. 166. 
of the above system are independent. If we assume that this is the case for $n=k-I$, the matrix $M_{k-1}$ of the system (which we may call $S_{k-1}$ ) has at least one determinant of order $k^{2}+1$ which does not vanish. Among the new rows and columns in $S_{k}$ is found the matrix

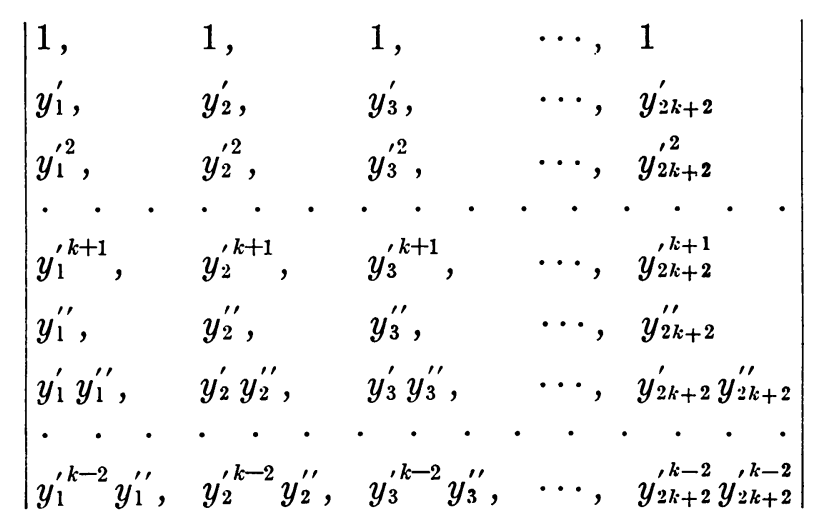

no one of whose $(2 k+1)$-rowed determinants vanishes. Thus $S_{k}$ has at least one $[k(k+2)+2]$-rowed determinant which does not vanish. If then, the system is independent for $n=k-1$, it is independent also for $n=k$. It is known to be so for $n=2$; hence the system is always independent. The number of independent solutions is $n^{2}$. Now four curves have an invariant of the first order, therefore the total number of invariants of the first order is $(2 n+2)-3$. Those of the second order independent of each other and of those of the first order number $2 n+2-5$, and so on; adding together all the essential invariants of orders 1 to $n-1$ we get $n^{2}-1$. The remaining invariant is of order $n$. We assert then the following

TheOREM. The smallest number of curves having contact of zero order possessing an invariant of order $n$ under the entire group of point transformations is $2 n+2$.

This agrees with the known results for $n=1, n=2$, given by Kasner.*

The area-preserving group is defined by the equation

$$
\xi_{x}+\eta_{y}=0 \text {. }
$$

By differentiating this equation we obtain two equations of the second order, viz.,

$$
\xi_{x x}+\eta_{x y}=0, \quad \xi_{x y}+\eta_{y y}=0 .
$$

The relation (9) is without effect in reducing the number of equations in the complete system for the whole group of point transformations extended to the first order, for $\xi_{x}$ and $\eta_{y}$ occur only in the expression $\eta_{y}-\xi_{x}$. Similarly, the equations (10) have no effect in reducing the complete system extended

\footnotetext{
${ }^{*}$ Loc. cit.
} 
to the second order. Since, however, $\xi_{x}$ and $\eta_{y}$ now occur in the combination $\eta_{y}-2 \xi_{x}$ which is independent of $\eta_{y}-\xi_{x}$, the equation (9) reduces the number of equations by 1 . In general, the $r$ equations of order $r$ obtained from (9) by differentiation will not affect the complete system for the whole group extended to the $r$ th order, but will reduce the system extended to the $(r+1)$ th order by $r$ equations. The complete system of the area-preserving group contains therefore $(1+2+3+\cdots+\overline{n-1})$ equations fewer than that of the entire group. The number of equations is therefore $\frac{1}{2} n(n+5)$. The formula (8) for $\lambda_{n}$ then gives $n+3$. Hence

TheOREM. The smallest number of curves having contact of zero order possessing an invariant of order $n$ under the area-preserving group is $n+3$.

The first type of invariant is obviously

$$
I=\frac{\left(y_{1}^{\prime}-y_{2}^{\prime}\right)\left(y_{3}^{\prime}-y_{4}^{\prime}\right)}{\left(y_{2}^{\prime}-y_{3}^{\prime}\right)\left(y_{4}^{\prime}-y_{1}^{\prime}\right)}
$$

which is the same as that of the entire group. We shall obtain the second by integrating the complete system. We have 7 equations in 10 variables:

$$
\begin{gathered}
U_{1} f \equiv \sum \frac{\partial f}{\partial y_{\imath}^{\prime}}=0 ; \quad U_{2} f \equiv \sum\left(2 y_{\imath}^{\prime} \frac{\partial f}{\partial y_{\imath}^{\prime}}+3 y_{\imath}^{\prime \prime} \frac{\partial f}{\partial y_{\imath}^{\prime \prime}}\right)=0 ; \\
U_{3} f \equiv \sum\left(y_{\imath}^{\prime 2} \frac{\partial f}{\partial y_{\imath}^{\prime}}+3 y_{\imath}^{\prime} y_{\imath}^{\prime \prime} \frac{\partial f}{\partial y_{\imath}^{\prime \prime}}\right)=0 ; \quad U_{4} f \equiv \sum \frac{\partial f}{\partial y_{\imath}^{\prime \prime}}=0 ; \\
U_{5} f \equiv \sum y_{\imath}^{\prime} \frac{\partial f}{\partial y_{\imath}^{\prime \prime}}=0 ; \quad U_{6} f \equiv \sum y_{\imath}^{\prime 2} \frac{\partial f}{\partial y_{\imath}^{\prime \prime}}=0 ; \quad U_{7} f \equiv \sum y_{\imath}^{\prime 3} \frac{\partial f}{\partial y_{\imath}^{\prime \prime}}=0,
\end{gathered}
$$

the summations extending from $i=1$ to $i=5$. By inspection we obtain five independent solutions of $U_{1}=U_{4}=U_{5}=U_{6}=U_{7}=0$, namely,

$$
Z_{3}=y_{1}^{\prime}-y_{j}^{\prime} \quad(j=2,3,4,5), \quad D_{5} \equiv\left|\begin{array}{l}
1, y_{1}^{\prime}, y_{1}^{\prime 2}, y_{1}^{\prime 3}, y_{1}^{\prime \prime} \\
1, y_{2}^{\prime}, y_{2}^{\prime 2}, y_{2}^{\prime 3}, y_{2}^{\prime \prime} \\
1, y_{3}^{\prime}, y_{3}^{\prime 2}, y_{3}^{\prime 3}, y_{3}^{\prime \prime} \\
1, y_{4}^{\prime},{y_{4}^{\prime}}^{2}, y_{4}^{\prime 3}, y_{4}^{\prime \prime} \\
1, y_{5}^{\prime}, y_{5}^{\prime 2}, y_{5}^{\prime 3}, y_{5}^{\prime \prime}
\end{array}\right| .
$$

Substituting in $U_{2}$ and $U_{3}$ we get

$$
\begin{gathered}
\sum_{j=2}^{5} 2 Z_{j} \frac{\partial f}{\partial Z_{j}}+15 D_{5} \frac{\partial f}{\partial D_{5}}=0 \\
\sum_{j=2}^{5}\left(y_{1}^{\prime}+y_{j}^{\prime}\right) Z_{j} \frac{\partial f}{\partial Z_{j}}+3 \sum_{i=1}^{5} y_{\imath}^{\prime} D_{5} \frac{\partial f}{\partial D_{5}}=0
\end{gathered}
$$


Multiplying equation (11) by $y_{1}^{\prime}$ and subtracting from (12)

$$
\sum_{j=2}^{5} Z_{j} \frac{\partial f}{\partial Z_{j}}+3 \sum_{j=2}^{5} Z_{j} D_{5} \frac{\partial f}{\partial D_{5}}=0 .
$$

We obtain four independent solutions of (13), viz.,

$$
\bar{Z}_{k} \equiv \frac{1}{Z_{2}}-\frac{1}{Z_{k}}(k=3,4,5), \quad \bar{D}=\frac{D_{5}^{1 / 3}}{Z_{2} Z_{3} Z_{4} Z_{5}} .
$$

Substituting in (11) we have

$$
2 \sum_{k=3}^{5} Z_{k} \frac{\partial f}{\partial \bar{Z}_{k}}+3 \bar{D} \frac{\partial f}{\partial \bar{D}}=0
$$

Finally, three independent solutions of (14) are

$$
\frac{\bar{Z}_{3}}{\bar{Z}_{5}}, \bar{Z}_{4}, \frac{\bar{D}_{5}^{1 / 3}}{\bar{Z}_{3}^{1 / 2}} \text {. }
$$

The first two are of the type $I$; when raised to the 9 th power and combined with the other two the third reduces to the form

$$
J_{5}=\frac{D_{5}^{2 / 3}\left(y_{1}^{\prime}-y_{2}^{\prime}\right)}{\left(y_{1}^{\prime}-y_{4}^{\prime}\right)^{2}\left(y_{1}^{\prime}-y_{5}^{\prime}\right)^{2}\left(y_{2}^{\prime}-y_{3}^{\prime}\right)^{3}} .
$$

Reverting for a moment to the complete system for the entire group, we notice that it consists of 8 equations -6 being identical with those of the area-preserving group, but the other two

$$
\begin{aligned}
& V_{1} f \equiv \sum y_{\imath}^{\prime} \frac{\partial f}{\partial y_{\imath}}=0, \\
& V_{2} f \equiv \sum y_{\imath}^{\prime \prime} \frac{\partial f}{\partial y_{\imath}^{\prime \prime}}=0
\end{aligned}
$$

being replaced in the area-preserving group by the single equation

$$
\bar{U}_{2} f \equiv\left(2 V_{1}+3 V_{2}\right) f=0,
$$

the summations now extending from 1 to 6 , a change which we will indicate by the bar over the symbol Uf. Now any solution of $\bar{U}_{2} f=0$ which is homogeneous and of degree zero both in the $y^{\prime \prime}$ and the $y^{\prime \prime}$ 's will be a solution of both $V_{1} f=0$ and $V_{2} f=0$. Now, we know two solutions of $\bar{U}_{2} f=0$, viz., $J_{5}$ and $J_{6}$, where $J_{6}$ is obtained from $J_{5}$ by the substitution of the subscript 6 for the subscript 5 . These also satisfy the remaining equations of the complete system. A function of $J_{5}$ and $J_{6}$ fulfilling all the required con- 
ditions is

$$
\left(\frac{J_{5}}{J_{6}}\right)^{3} \equiv \frac{\left(y_{1}^{\prime}-y_{6}^{\prime}\right)^{3} D_{5}}{\left(y_{1}^{\prime}-y_{5}^{\prime}\right)^{3} D_{6}},
$$

$D_{6}$ being obtained from $D_{5}$ by the substitution of the subscript 6 for the subscript 5. This is the type of invariant obtained by Kasner*-though in a different form-which showed the inadequacy of Rabut's treatment of the entire group of point transformations.

The invariants of the entire group are always homogeneous of degree zero in all of the variables (though not necessarily in the variables of each order). This appears from the fourth equation of the complete system. The fifth equation shows that the weight of every invariant is zero, a result stated by Rabut. In the area-preserving group these two equations are replaced by the single equation

$$
\sum 2 y_{\imath}^{\prime} \frac{\partial f}{\partial y_{\imath}^{\prime}}+3 y_{\imath}^{\prime \prime} \frac{\partial f}{\partial y_{\imath}^{\prime \prime}}+\cdots+(n+1) y_{\imath}^{(n)} \frac{\partial f}{\partial y_{\imath}^{(n)}}=0
$$

which has a set of fundamental solutions

$$
y_{i}^{\prime 1 / 2} \div\left(y_{j}^{(k)}\right)^{1 /(k+1)}
$$

showing that the following theorem is true.

THEOREM. If the order of derivation of every variable occurring in an invariant of the area-preserving group be increased by unity, the result is an expression of weight zero.

(After the above operation, both numerator and denominator of $J_{5}$ are of weight 14.)

The factors $y_{\imath}^{\prime}-y_{j}^{\prime}$ and $D_{5}$ occurring in the expressions $J_{5}$ and $J_{5} \div J_{6}$ are relative invariants of the entire group. The magnifying factor of the first is

while that of $D_{5}$ is

$$
\frac{\Phi_{x} \Psi_{y}-\Psi_{x} \Phi_{y}}{\left(\Phi_{x}+\Phi_{y} y_{\imath}^{\prime}\right)\left(\Phi_{x}+\Phi_{y} y_{j}^{\prime}\right)}
$$

$$
\frac{\left(\Phi_{x} \Psi_{y}-\Psi_{x} \Phi_{y}\right)^{7}}{\prod_{\imath=1}^{i=5}\left(\Phi_{x}+\Phi_{y} y_{\iota}^{\prime}\right)^{3}}
$$

In the case of the area-preserving group $\Phi_{x} \Psi_{y}-\Psi_{x} \Phi_{y}=1$.

We give a third invariant of this latter group, which was obtained by the method described in the next section. A convenient form is

*P. 210. 


$$
K_{6}=\frac{\left[\begin{array}{lll}
1 & 2 & 3 \\
1 & 2 & 3
\end{array}\right]^{2}}{\left[\begin{array}{ll}
1 & 2 \\
1 & 2
\end{array}\right]^{4}\left[\begin{array}{ll}
1 & 3 \\
1 & 2
\end{array}\right]^{4}}
$$

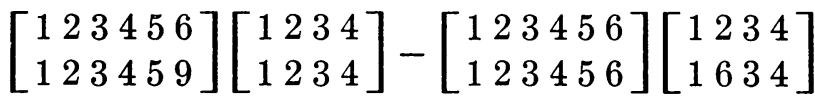

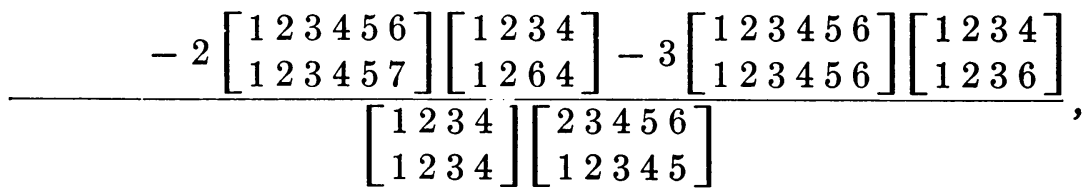

where the quantities in brackets are determinants formed from the matrix whose $i$ th row is the following:

$$
1 \quad y_{\imath}^{\prime} \quad y_{\imath}^{\prime 2} y_{\imath}^{\prime 3} y_{\imath}^{\prime 4} y_{\imath}^{\prime \prime} y_{\imath}^{\prime} y_{\imath}^{\prime \prime} y_{\imath}^{\prime 2} y_{\imath}^{\prime \prime} y_{\imath}^{\prime \prime \prime},
$$

the upper and lower rows of numerals within each bracket indicating respectively the rows and columns of the matrix from which the determinant is constructed.

From this result we can build up a third-order invariant of the entire group for 8 curves. The complete system equivalent to the entire group contains the equations

$$
\begin{aligned}
& W_{1} f=\sum\left(y_{\imath}^{\prime} \frac{\partial f}{\partial y_{\imath}^{\prime}}+y_{\imath}^{\prime \prime} \frac{\partial f}{\partial y_{\imath}^{\prime \prime}}+y_{\imath}^{\prime \prime \prime} \frac{\partial f}{\partial y_{\imath}^{\prime \prime \prime}}\right)=0, \\
& W_{2} f=\sum y_{\imath}^{\prime \prime} \frac{\partial f}{\partial y_{\imath}^{\prime \prime \prime}}=0, \\
& W_{3} f=\sum y_{\imath}^{\prime} y_{\imath}^{\prime \prime} \frac{\partial f}{\partial y_{\imath}^{\prime \prime \prime}}=0,
\end{aligned}
$$

in addition to the twelve whose solutions we have found.

The configuration has three independent solutions of the third order under the area-preserving group, viz.,

$$
K_{6}, K_{7}, K_{8}
$$

where $K_{7}, K_{8}$ are obtained from $K_{6}$ by the substitution of the subscripts 7 and 8 respectively for the subscript 6 . Let us seek a linear function of $K_{6}, K_{7}, K_{8}$ which shall satisfy the equations $W_{2} f=0$ and $W_{3} f=0$. If

$$
A_{6} K_{6}+A_{7} K_{7}+A_{8} K_{8} \text {, }
$$

be such a function ( $A_{6}, A_{7}, A_{8}$ being of the second order at most), we must 
have

$$
\begin{aligned}
& A_{6} W_{2}\left(K_{6}\right)+A_{7} W_{2}\left(K_{7}\right)+A_{8} W_{2}\left(K_{8}\right)=0, \\
& A_{6} W_{3}\left(K_{6}\right)+A_{7} W_{3}\left(K_{7}\right)+A_{8} W_{3}\left(K_{8}\right)=0 .
\end{aligned}
$$

Hence $A_{6}, A_{7}, A_{8}$ must be proportional to three quantities of which the first is

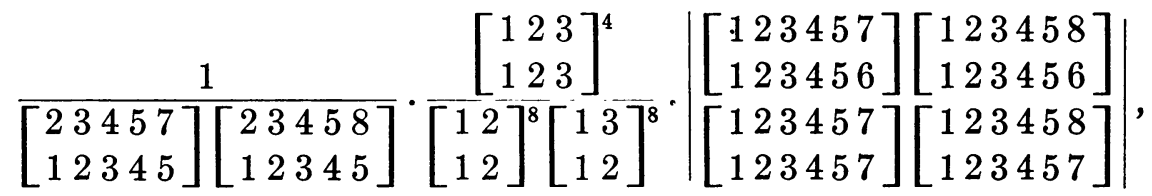

and the others are obtained from this by a cyclic interchange oi the subscripts 6,7 , and 8 . Now under the entire group the magnifying factors of the relative invariants

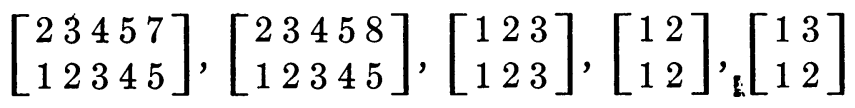

are respectively

$$
\begin{aligned}
& \frac{\left(\Phi_{x} \Psi_{y}-\Psi_{x} \Phi_{y}\right)^{10}}{\prod_{\imath=23457}\left(\Phi_{x}+\Phi_{y} y_{\imath}^{\prime}\right)^{4}}, \quad \frac{\left(\Phi_{x} \Psi_{y}-\Psi_{x} \Phi_{y}\right)^{10}}{\prod_{\imath=23458}\left(\Phi_{x}+\Phi_{y} y_{\imath}^{\prime}\right)^{4}}, \quad \frac{\left(\Phi_{x} \Psi_{y}-\Psi_{x} \Phi_{y}\right)^{3}}{\prod_{\iota=123}\left(\Phi_{x}+\Phi_{y} y_{\imath}^{\prime}\right)^{2}}, \\
& \frac{\Phi_{x} \Psi_{y}-\Psi_{x} \Phi_{y}}{\prod_{12}\left(\Phi_{x}+\Phi_{y} y_{\imath}^{\prime}\right)}, \quad \frac{\Phi_{x} \Psi_{y}-\Psi_{x} \Phi_{y}}{\prod_{13}\left(\Phi_{x}+\Phi_{y} y_{\imath}^{\prime}\right)}
\end{aligned}
$$

The four 6-rowed determinants are not relative invariants, but

become respectively

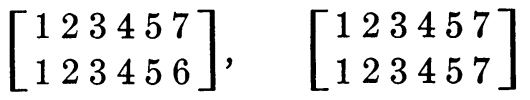

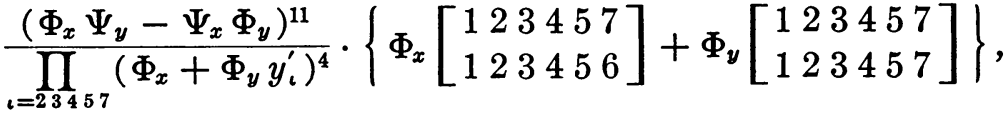

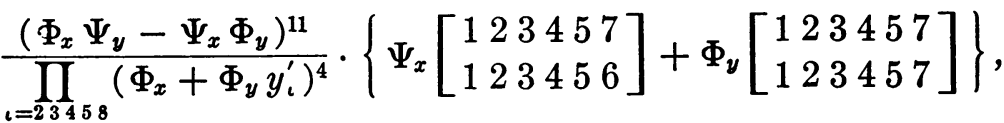

and the other two similar expressions are obtained by substituting the subscript 8 for the subscript 7 .

The determinant of the last four is therefore a relative invariant with the magnifying factor

$$
\frac{\left(\Phi_{x} \Psi_{y}-\Psi_{x} \Phi_{y}\right)^{23}}{\left(\prod_{123457}\right)\left(\prod_{12345}\right)}
$$


The whole expression (15) is found on multiplication to be a relative invariant of the entire group with the magnifying factor $\left(\Phi_{x} \Psi_{y}-\Psi_{x} \Phi_{y}\right)^{-1}$, and this holds likewise for the two similar expressions. If, then, we take $A_{6}, A_{7}, A_{8}$ equal-and not merely proportional-to the expressions of type (15), we see that

$$
{ }^{A_{6}} K_{6}+A_{7} K_{7}+A_{8} K_{8}
$$

is an absolute invariant of the area-preserving group. It satisfies, moreover, the equations $W_{2} f=0, W_{3} f=0$.

There remains only the equation $W_{1} f=0$; this shows that our invariant must be homogeneous of degree zero in all of the variables. The expression (16) is homogeneous of degree $-2 . J_{5}$ is an absolute invariant of the areapreserving group, and satisfies the equations $W_{2} f=0, W_{3} f=0$. It is homogeneous and of degree $-\frac{1}{3}$. The expression

$$
J_{5}^{-6}\left(A_{6} K_{6}+A_{7} K_{7}+A_{8} K_{8}\right)
$$

is therefore homogeneous of degree zero, and is a third-order invariant of the entire group of point transformations.

We do not wish to enter here into the question of what we may regard as limiting forms of the configurations we have just considered, i. e., those configurations some of whose elements have contact of higher than zero order. Rabut has discussed a certain class of them for the group of all point transformations. There are, however, limiting configurations even of the second order possessing invariants under this group which he has failed to detect.

6 . The equilong group. The imprimitive group

$$
X=\Phi(x), \quad Y=y \Phi^{\prime}(x)+\chi(x)
$$

is known as the equilong group of transformations* when $x$ and $y$ are interpreted as Hessian line coördinates, and has interesting geometric properties. $\dagger$

We confine ourselves to point coördinates. It is clear from the result of Section 4 that three elements having the same abscissa but different ordinates for the points at which they are situated have an infinite series of invariants.

In the consideration of this group we shall depart from the method of infinitesimal transformations; it is a little more convenient to use the method of elimination. $\ddagger$ This process consists in successive differentiation of the finite equations of the group, and elimination from the resulting equations of the arbitrary functions. A relation of the $n$th order of the form

$$
f\left(x, y_{\imath}, y_{\iota}^{\prime}, \cdots, y_{\iota}^{(n)} ; X, Y_{\iota}, Y_{\iota}^{\prime}, \cdots, Y_{\iota}^{(n)}\right)=0
$$

* Scheffers, Mathematis che Annalen, vol. 60 .

$\dagger$ See papers by Kasner already cited, and also P. H. Linehan, Contributions to equilong geometry, Dissertation, Columbia University (1915).

$\ddagger$ Lie, Theorie der Transformationsgruppen, vol. 1, Kap. 13, §58. 
being obtained, the separation of the variables is effected by replacing all the variables but one of the transformed configuration by constants, and then solving for the remaining one. The result is an invariant function of the variables of the first configuration. Care must be taken in the choice of the constants to see that the configuration corresponding to the chosen values has no special properties under the group.

To carry out the operations we have indicated, it is convenient to choose the equations in the form

$$
X=\int e^{-\Phi(x)} d x, \quad Y=y e^{-\Phi(x)}+\chi_{1}(x) .
$$

Differentiating $n$ times in succession we get

$$
\begin{aligned}
Y^{\prime} & =y^{\prime}-\Phi^{\prime} y+\chi_{2}, \\
Y^{\prime \prime} & =e^{\Phi}\left\{y^{\prime \prime}-\left(\Phi^{\prime} y^{\prime}+\Phi^{\prime \prime} y\right)\right\}+\chi_{3}, \\
\cdot & \cdot \cdot \cdot \cdot \cdot \cdot \cdot \cdot \cdot \cdot \cdot, \\
Y^{(n)} & =e^{\Phi} \frac{d}{d x}\left(Y^{(n-1)}\right) .
\end{aligned}
$$

The substitution of the constants for $Y_{\imath}, Y_{\imath}, \cdots, Y_{\imath}^{(n)}$ may be conveniently performed before the elimination of the arbitrary functions. We choose $Y_{1}-Y_{2}=1, Y_{1}^{(k)}-Y_{2}^{(k)}=0$ for all values of $k$ from 1 to $n$. This restriction on two of the elements of the transformed configuration leaves that configuration perfectly general under the group. We have to solve the following equations for the arbitrary functions $e^{\Phi}, \Phi^{\prime}, \Phi^{\prime \prime}, \cdots, \Phi^{(n)}$.

$$
\begin{aligned}
& 1=e^{-\Phi}\left(y_{1}-y_{2}\right), \\
& 0=y_{1}^{\prime}-y_{2}^{\prime}-\Phi^{\prime}\left(y_{1}-y_{2}\right) \text {, } \\
& 0=y_{1}^{\prime \prime}-y_{2}^{\prime \prime}-\Phi^{\prime}\left(y_{1}^{\prime}-y_{2}^{\prime}\right)-\Phi^{\prime \prime}\left(y_{1}-y_{2}\right) \text {, } \\
& 0=y_{1}^{(n)}-y_{2}^{(n)}-\Phi^{\prime}\left(y_{1}^{(n-1)}-y_{2}^{(n-1)}\right)-c_{1} \Phi^{\prime \prime}\left(y_{1}^{(n-2)}-y_{2}^{(n-2)}\right)-\cdots .
\end{aligned}
$$

A great advantage of this particular choice of the constants now appears. Each of the equations (17) might be obtained by direct differentiation from the preceding, therefore when we have expressed $e^{\Phi}, \Phi^{\prime}, \cdots, \Phi^{(n)}$ in terms of $y_{\imath}^{(k)}-y_{2}^{(k)}$ by solving these equations we see that $\Phi^{\prime \prime}, \Phi^{\prime \prime \prime}, \ldots, \Phi^{(n)}$ are such as follow from $\Phi^{\prime \prime}$ by successive differentiation, and that $\Phi^{\prime} e^{\Phi}$ is simply the derivative of $e^{\Phi}$. We have then

$$
e^{\Phi}=y_{1}-y_{2}, \quad \Phi^{\prime}=\frac{y_{1}^{\prime}-y_{2}^{\prime}}{y_{1}-y_{2}}, \quad \Phi^{\prime \prime}=\frac{d \Phi^{\prime}}{d x}, \quad \cdots .
$$

We have to substitute these values successively in the equations 


$$
\begin{aligned}
& Y_{1}-Y_{3}=e^{-\Phi}\left(y_{1}-y_{3}\right), \\
& Y_{1}^{\prime}-Y_{3}^{\prime}=y_{1}^{\prime}-y_{3}^{\prime}-\Phi^{\prime}\left(y_{1}-y_{3}\right) \text {, } \\
& Y_{1}^{\prime \prime}-Y_{3}^{\prime \prime}=e^{\Phi}\left\{y_{1}^{\prime \prime}-y_{3}^{\prime \prime}-\Phi^{\prime}\left(y_{1}^{\prime}-y_{3}^{\prime}\right)-\Phi^{\prime \prime}\left(y_{1}-y_{3}\right)\right\} \text {, } \\
& Y_{1}^{(n)}-Y_{3}^{(n)}=e^{\Phi} \frac{d}{d x}\left(Y_{1}^{(n-1)}-Y_{3}^{(n-1)}\right) .
\end{aligned}
$$

The expressions on the right are the invariants of the different orders. But from the way in which the constants were chosen we see that the operation by which the equations (17) are successively derived might just as well be performed after the substitution of the values (18) as before. In general, then

$$
I_{k}=\left(y_{1}-y_{2}\right) \frac{d I_{k-1}}{d x},
$$

where $I_{k}$ is the invariant of order $k$, while $I_{k-1}$ is that of order $k-1$. The first of the series is

$$
I_{0} \equiv \frac{y_{1}-y_{3}}{y_{1}-y_{2}}
$$

\section{Contact transformations}

1. Finite groups. Since " contact of zero order" is not preserved by contact transformations in general, no distinction can be made between configurations whose elements have contact of zero order and those whose elements are situated at different neighboring points. With this exception, the results obtained for finite groups of point transformations hold also for finite groups of contact transformations. The fundamental configuration consists of two elements through two distinct points $\left(x_{1} y_{1}\right)$ and $\left(x_{2} y_{2}\right)$; and the number of independent mixed invariants is equal to the number of essential parameters in the group.

2. Infinite groups. Single element. In the case of infinite groups single elements may have invariants of order zero or 1 . If an invariant of order zero exists, $f(x, y)$ say, we have

$$
\Omega(x, y, X, Y) \equiv f(X, Y)-f(x, y)=0 .
$$

Then the determinant

$$
\left|\begin{array}{lll}
0, & \frac{\partial \Omega}{\partial x}, & \frac{\partial \Omega}{\partial y} \\
\frac{\partial \Omega}{\partial X}, & \frac{\partial^{2} \Omega}{\partial x \partial X}, & \frac{\partial^{2} \Omega}{\partial y \partial X} \\
\frac{\partial \Omega}{\partial Y}, & \frac{\partial^{2} \Omega}{\partial x \partial Y}, & \frac{\partial^{2} \Omega}{\partial y \partial Y}
\end{array}\right|
$$


vanishes identically. We have then the

Theorem. A contact transformation having an invariant of order zero is merely an extended point transformation.

All groups of contact transformations leaving invariant a differential equation of the second order are reducible to the group of point transformations. Hence the

TheOREM. A contact transformation having an invariant of the first order is reducible to the form

$$
X=x, \quad Y=\Psi(x, y) .
$$

Finally, we know that no infinite group of contact transformations can leave invariant a differential equation of the third or higher order, and we have the

THEOREM. No infinite group of contact transformations can have an invariant of a single element of the second or higher order.

3. Infinite groups. Rabut's Theorem. Every infinite group of contact transformations has, however, invariants of certain configurations. The group of all contact transformations was treated by Rabut, who showed that it had no essential invariants other than those of individual bunches, and, moreover, that all the elements must have contact of at least the first order. Under these circumstances he proved the

TheOREM. The invariants of the group of all contact transformations are simply those of the group of all point transformations, except that the order of each derivative is increased by unity.

We shall verify this theorem by comparing the equivalent complete systems of the two groups.

The general infinitesimal transformation of the group of all contact transformations is

$$
\delta x=\xi\left(x, y, y^{\prime}\right) \delta t, \quad \delta y=\eta\left(x, y, y^{\prime}\right) \delta t, \quad \delta y^{\prime}=\pi\left(x, y, y^{\prime}\right) \delta t,
$$

where $\xi, \eta, \pi$ are independent functions of $x, y, y^{\prime}$ expressible, however, in terms of the derivatives of one arbitrary function $W\left(x, y, y^{\prime}\right)$. We have

$$
\xi=W_{y^{\prime}}, \quad \eta=y^{\prime} W_{y^{\prime}}-W, \quad \pi=-W_{x}-W_{y} y^{\prime} .
$$

The increment of $y^{\prime \prime}$ is

$$
\eta^{\prime \prime}=\left(\pi_{x}+\pi_{y} y^{\prime}\right)+y^{\prime \prime}\left\{\pi_{y^{\prime}}-\left(\xi_{x}+\xi_{y} y^{\prime}\right)\right\}-y^{\prime \prime^{2}} \xi_{y^{\prime}} .
$$

Compare this with the increment of $y^{\prime}$ under the group of all point transformations. The rule of operation for the successive increments is always for $n>1$

$$
\eta^{(n)}=\frac{d \eta^{(n-1)}}{d x}-y^{(n)} \frac{d \xi}{d x},
$$


exactly as in the case of the point transformations. The only difference is that instead of the arbitrary functions of two variables $f(x, y), f_{x}(x, y)$, $f_{y}(x, y)$, we now have $F\left(x, y, y^{\prime}\right), F_{x}\left(x, y, y^{\prime}\right)+y^{\prime} F_{y}\left(x, y, y^{\prime}\right)$, and $F_{y^{\prime}}\left(x, y, y^{\prime}\right)$, and that $y^{(k+1)}$ always takes the place of $y^{(k)}$. We thus get the same complete system for order $n+1$ as in the case of the point transformations for order $n$, except that $y^{(k+1)}$ takes the place of $y^{(k)}$. We find that $2 n+2$ curves have an invariant of order $n+1$, obtained from the corresponding invariant of order $n$ for the point transformations by the above substitution.

4. Certain reducible groups. We noticed an exception to the general rule that each bunch of elements had its own invariants in the case of infinite groups of point transformations. A similar exception occurs in the case of a certain class of contact transformations, viz., those which leave invariant a differential equation of the second order in a special way: if $u\left(x, y, y^{\prime}\right)$ be a first integral, they transform the $\infty^{1}$ families $u=c$ among themselves. If we choose such a configuration that the first three coördinates $x, y, y^{\prime}$ of each element satisfy a relation $u\left(x, y, y^{\prime}\right)=c$, that is, the elements have firstorder contact with different curves of the same family of integral-curves, the function $u\left(x, y, y^{\prime}\right)$ will be the same for each bunch. An arbitrary function $\lambda(u)$ occurring in the extended infinitesimal transformations of the group will lead to equations in the equivalent complete system among variables from all the bunches. It is sometimes possible under these circumstances for the configuration to have invariants other than those of individual bunches.

It should be noticed that if the function $u$ does not involve $y^{\prime}$, the group which leaves the totality of the $\infty^{1}$ families $u=c$ invariant is merely a group of point transformations. For we have a relation of the type

$$
\Omega(x, y, X, Y) \equiv u(X, Y)-f\{u(x, y)\}=0,
$$

which is such that the determinant (1) vanishes.

Now the group of contact transformations which leaves invariant a given differential equation of the second order is reducible to the group of point transformations. All such groups are of the form

$$
\begin{aligned}
& u\left(X, Y, Y^{\prime}\right)=\Phi\left\{u\left(x, y, y^{\prime}\right), v\left(x, y, y^{\prime}\right)\right\}, \\
& v\left(X, Y, Y^{\prime}\right)=\Psi\left\{u\left(x, y, y^{\prime}\right), v\left(x, y, y^{\prime}\right)\right\},
\end{aligned}
$$

where $u$ and $v$ are independent first integrals of the given differential equation. If the group has the special property of leaving the totality of the $\infty^{1}$ families $u=c$ invariant, it has the form

$$
\begin{aligned}
& u\left(X, Y, Y^{\prime}\right)=\Phi\left\{u\left(x, y, y^{\prime}\right)\right\} \\
& v\left(X, Y, Y^{\prime}\right)=\Psi\left\{u\left(x, y, y^{\prime}\right), v\left(x, y, y^{\prime}\right)\right\} .
\end{aligned}
$$


If we transform this group by the contact transformation

$$
\begin{aligned}
& x=u\left(x, y, y^{\prime}\right), \\
& y=v\left(x, y, y^{\prime}\right),
\end{aligned}
$$

we obtain the group of point transformations

$$
\begin{aligned}
& X=\Phi(x), \\
& Y=\Psi(x, y) .
\end{aligned}
$$

It is now clear that the only groups possessing essential invariants of more than one bunch of elements are those for which $\Psi(u, v)$ has the special form

$$
\frac{\Psi_{1}(u)+v \Psi_{2}(u)}{\Psi_{3}(u)+v \Psi_{4}(u)} .
$$

These are all reducible by the transformation (2) to the group of point transformations of Section 3, Part II. They have an invariant of the first order of four elements

$$
\frac{\left(v_{1}-v_{2}\right)\left(v_{3}-v_{4}\right)}{\left(v_{2}-v_{3}\right)\left(v_{4}-v_{1}\right)}
$$

and thereafter a series of invariants, one of each order, for five curves.

A simple illustration is the largest subgroup of the group preserving parallelism. Here $u=y^{\prime}, v=y-x y^{\prime}$, and the group has the form

$$
\begin{aligned}
Y^{\prime} & =\Phi\left(y^{\prime}\right), \\
Y-X Y^{\prime} & =\frac{\Psi_{1}\left(y^{\prime}\right)+\Psi_{2}\left(y^{\prime}\right) \cdot\left(y-x y^{\prime}\right)}{\Psi_{3}\left(y^{\prime}\right)+\Psi_{4}\left(y^{\prime}\right) \cdot\left(y-x y^{\prime}\right)} .
\end{aligned}
$$

This is reduced to a group of point transformations by the contact transformation

$$
\begin{aligned}
X & =y^{\prime}, \\
Y & =y-x y^{\prime}, \\
Y^{\prime} & =-x .
\end{aligned}
$$

The group has the invariant of four parallel elements

$$
\frac{\left\{y_{1}-y_{2}+\left(x_{1}-x_{2}\right) y^{\prime}\right\}\left\{y_{3}-y_{4}+\left(x_{3}-x_{4}\right) y^{\prime}\right\}}{\left\{y_{2}-y_{3}+\left(x_{2}-x_{3}\right) y^{\prime}\right\}\left\{y_{4}-y_{1}+\left(x_{4}-x_{1}\right) y^{\prime}\right\}}
$$

Five elements whose tangents are parallel have a series of invariants one of each order $n>1$.

5. Number of elements having invariants. Finally, the argument of Section 4, Part II, is applicable to contact transformations, and we obtain the 
THEOREM. If the group involves arbitrary functions of more than one variable, $\lambda_{n}$ is an increasing function of $n$; otherwise it attains a final value $k+1$ (where $k$ is the number of arbitrary functions of one variable in the unextended form of the group), after a certain finite value of $n$. 